\title{
Archives, Arcades, and the Translation of Neologisms
}

PROBAL DASGUPTA*

For convenience, here is a summary of the expository sequence in this paper. Section 1 observes that neologisms are nondomesticated new expressions, and that translators responding to the problem of tackling neologisms often need to go back to the basics and develop an overall understanding of the issue of newness: The issue is fundamental since a translation must both be new to the TL (and meet a felt need for something missing in the TL) and keep in touch with old trends in the TL (so that normal TL readers understand the translation and do not find it opaquely foreign).

Section 2 on domains and innovative styles shows that all domains of discourse have their own technical details that keep evolving, producing novelties for the translators in that field to keep up with. The way this happens varies from domain to domain.

Section 3 addresses the question of why abbreviations become so frequent in all domains of the modern use of language in contrast to classical societies. It is argued that this happens because the modern period brings with it an overriding interest in saving time and in abbreviating all processes.

Section 4 argues that modernity prizes originality, that original creators wish to mark their work with special identifiers, that expressions serving in this role become name-like, that names need to be entered in registers in some sense, that registers in traditional societies tended to emanate from a single monarchical source of power and thus to belong to a single central archive, and that, in contrast, the typical registers in modern societies are the decentralized body of newspapers which hold and disseminate public information.

\footnotetext{
"Probal Dasgupta is a Professor in the Center of Applied Linguistics and Translation Studies, University of Hyderabad, Hyderabad. He is reachable at pdgalts@uohyd.ernet.in
} 
Section 5 notes that the arcades of newspaper-borne and other advertisements of decentralized originality do not, in modern social formations, quite supersede the traditional archives which modernity retains. But they prevail in modern societies. This creates a nonisomorphism between the body of records and therefore of resonances in a traditional society and the corresponding set of resonances in a modern formation, posing acute difficulties for a translator working at the interface between a traditional and a modern language.

Section 6, on Less Equipped Languages or LELs, proposes that the problem is solved by the expectant speech communities of LELs who are waiting for imported innovations, among other things, to sweep them off their traditional feet. This section lists certain types of neologism and suggests practical strategies for facing the different problems they pose for translating from a MEL to LEL.

The seventh and the last section suggests that journalists and educators are the strongest forces implementing language modernization, and that they mediate between the public and the language planning authorities. But language planners are generally too conservative to cope with the pace and type of innovations now in progress, for structural reasons having to do with the composition of the relevant planning committees in their world societies. Ways around this problem are pointed out.

\section{Introduction}

\subsection{Translation and Newness}

Neologisms are, at the most evident level, new words or expressions not yet regarded as completely naturalized or domesticated elements in the language. For instance, non-Russian journalists reporting the achievements of Mikhail Gorbachev in the eighties adopted the loanwords glasnost and perestroika in their respective languages to describe his brand of 'openness' and 'restructuring'. These neologisms spread rapidly and became part of the common terminological currency all over the world. But they are not ordinary words in our languages. They have remained neologisms. 
The problem takes on an added dimension in cases like sputnik, which in the 1950s was a new coinage in Russian itself - in contrast to the ordinary Russian words glasnost and perestroika. In the glasnost case, an ordinary SL term gives rise to neologisms in the TLs. But sputnik instantiates a different pattern. Here both the SL word and its copies in the TL are brand new. A neologism is translated by a neologistic loanword. However, the difference is not as big as it looks. Although glasnost and perestroika already existed in Russian, Gorbachev gave them a twist and "rrned them into his brand names for the new social technology was advertising. Thus, even in Russian, glasnost and perestroikc unt as 'semantic neologisms' (old words with the new meanings) in their Gorbachevian use which led to their massive adoption all over the world.

The above cases are prototypical examples. They help us to focus on the general problem of neologisms. The fundamental question is how translators can decide when to resort to neologisms in their work and what strategies to use when direct borrowing is not a viable option.

We can tackle this question effectively if we go about it with some circumspection. Our approach here is to explore the general issues of innovation in language in the context of translation.

\subsection{The Translation Novelty Paradox}

Consider the following paradox: Any TL document D translated from an SL must by definition be new to TL. If D already existed in TL, nobody would need to translate the material from an SL. But D must consist of pieces (i.e. words and expressions) which are old in TL, for it they do not count as common currency then the audience will not understand, defeating the whole purpose of translation. Thus, D must be new to TL, and yet must be composed of material that is old in TL.

The Translation Novelty Paradox, as we may call it for reference, reflects a contested area where two forces pull the translator in opposite directions. There is the need for $\mathrm{D}$ to preserve continuity with existing TL writings. This need propels us towards traditional, comfortable translations. But D also needs to perform a 
distinct rupture with the tradition and specifically make, room for the exact content of this text coming from outside - a need that encourages technical, accurate translations, capable of specifying discontinuities with some rigour.

The direction in which the stronger winds blow changes as we move from traditional to modern social formations. In traditional contexts, even the original SL text would have normally been written along prescribed lines, with little or no self-conscious innovation. And a traditional TL translation D naturally follows suit, departing minimally or not at all from the composition conventions in the TL culture. But in the modern period marked by the rise of the technical domains, much writing takes place under the shadow of what we shall call the technical contribution imperative. The principle demands that all serious statements must (a) contribute some new and original thinking self-consciously marked in the very form of the text, and (b) link this work to some general impersonal system of expressing thoughts and feelings with objectivity and precision. Both the particular technique of the individual writer and the systematic technology of the collective field of such writing make modern texts especially prone to exact and differentiated modes of expression. This often takes the form of neologisms in original writings as well as translations. For only innovations can sharpen the ordinary, diffuse language into a vehicle for the required level of exact expression.

Thus, authors and translators typically strove for an old, tradition-maintaining appearance in their writings in classical cultures. Correspondingly, most writers and rewriters strive to package their products as stimulatingly novel commodities in the modern market. Our approach will be to grasp this overall contrast in terms of the classical archive of traditional texts and translations versus the modern arcade of textual wares advertised as excitingly special, new, and definitely to be bought. The archive and the arcade present two different modes of putting texts together to build larger aggregates. We will now explore various dimensions of the archive/arcade conception of the novelty problem in translation studies. 


\section{Domains and Innovation Styles}

The problem of novelty shows up differently depending on the domain of language use involved. Fields like science, technology, business, law, public administration, management or medicine constantly come up with whole sets of new terms and usages. They all challenge the translator. But not in the same way. It is useful to notice some of the variations.

\subsection{Business and Law vs Science and Technology}

At the most obvious level, business and law are more conservative domains than science and technology. While business people deal with new merchandise all the time, they treat merchandise description terms as mere names to be placed in lists. The real business terms which make their discourse do not easily change. And the judiciary's tendency to preserve continuity and to resist change is of course well known. In contrast, the spirit of innovation is what keeps science and technology going. Practitioners of those fields have to propose new ideas and invent new methods and machines in order to survive. So they are compelled to use new terms reflecting the constant changes in their thinking.

\subsection{Business Language vs Advertisements}

Of course, business has to make use of technological innovations all the time. Any successful entrepreneur must exploit new technology to cut costs and beat the competition. Business success also depends on convincing the public that one is producing better wares than one's rivals are offering. This job of convincing the public is done in the field of advertising, which is not quite the same as the field of business proper for our purposes. Consider a newspaper. The business pages have an entirely different look to them, in organization and language, compared to the readerfriendly, graphically attractive advertisements. This shows that the language of advertising and the language of business pose entirely different challenges to the translator. Advertising is a field where linguistic novelty is a constant feature. Business discourse proper is not. When business people discuss technological innovations for reasons internal to technology rather than business. But that gives 
the translator headaches which can be solved only by consulting the relevant technological glossaries and reference works, not by a careful study of business language in general. This is what is meant by saying that business is linguistically a conservative domain.

\subsection{Innovations in Non-technological Domains}

However, this type of classification of domains is only superficial. It focuses on the frequency of use of new terms alone. The translator has to deal with novelty as a whole. Even.in terminologically conservative domains like business, there is a constant process throwing up new abbreviations for the translator to decipher. Correspondingly, legal language, even if it rests on a firm and slow-changing system of usage, naturally refers to the whole apparatus of laws and precedents constituting the systemic background presupposed in all legal discussion within a given country. Translators can cope with such material only if they know their way around the legal literature of that country. The problem is compounded in a nation like the United States of America, where much political reform takes place outside the legislature in the form of landmark judgments by the Supreme Court. For in such a country the normal political discourse of journalism is replete with references to specific cases, judgments and legal discussions.

Thus, in practice, even the non-technologically oriented domains have their own technical details which keep changing as the domain evolves, producing novelties for the translator in that field to keep up with. It is for this reason that translators have to acquire and keep refreshing some specialized disciplinary knowledge in addition to an overall command over the SL and the TL. They have to follow the field, keeping up with new developments in it on both sides of the SL-TL divide that they are trying to bridge. This is the only way to retain enough familiarity with the scene to make it possible to figure out the meaning of particular abbreviations or other new details under the real-life conditions of specialized translation, where time is a scarce resource and workers have to find answers to their puzzles very quickly. 


\section{Abbreviative Practices}

When we classify domains the way we did in section 2 , it may seem to us that some are more technical than others in some obvious sense. If we accept the view presented earlier about the role of the technical in the increased frequency of novelty in modern language use, it then follows that a field is going to be neologism- prone in proportion to its degree of technicality. But abbreviations pose a problem that cuts across domains. They are characteristic of modern languages in general. Classical languages used abbreviations very sparingly.

\subsection{Frequency of Abbreviations}

Why are abbreviations so frequent in all domains of the modern use of language? The most obvious reason is that modern life prizes time as a scarce and highly valuable resource. The use of abbreviations is a gesture marking our general impatience with the traditional method of using long-winded names and repeating them. In other words, the rise of abbreviation as a general social force reflects the same underlying factors as the industrial-technological imperative as a whole. We wish to save valuable time and then use the time for other purposes - to be chosen by relatively free human beings under conditions shaped by the new, labour-saving, technology-driven, abbreviative society. Paradoxically, the need to be in tune with one's time forces people to keep learning and relearning standard lists of abbreviations, losing some of the precious time one would have expected to save. This paradox, again, is a version of the general irony of a technology which tries to release people's energies for their individually chosen desires but which in practice binds people's tastes by promoting ruthless advertisement campaigns as a general commercial practice, turning nearly all new words into trademarks or brand names.

\subsection{Translators and Clarification}

Evidently, the translator is a person who tries to remove the language barrier and other obstructions preventing clear communication. It is thus our job, as clarifiers, to help demystify unnecessary jargon and pointless abbreviations. But "unnecessary" is the operative term here. Translators seek high fidelity. Modern 
translators live in a period when this is abbreviated as hi fi in the context of sound recording practices of the postwar period. This example shows that abbreviations, like other key words emblematic of a particular discursive domain, encode the domain itself and thus often function as indispensable markers of how the reader should situate what is being said. They serve to indicate the context and are not mere decorations which a clarity-seeking translator may remove at will. This is also true of other uses of technical language in passages intended for lay audiences. Translators may be abie to detect cases where the SL author is simply showing off or is choosing technical rather than lay language because of irrelevant factors like the inability to think of ordinary terms (some authors get used to their specialized jargon and become unable to shed that diction even when addressing a wider readership). In such instances, it is their privilege and even their duty to remove the extra technicality when they translate, including. opaque abbreviations. But very often the apparently unnecessary technical terms or specialized, context-bound abbreviations in fact perform the important task of telling the reader quickly what context is being invoked. Not only the author, but the reader too is in a hurry in the modern age. Thus, both parties to the transaction of hasty communication need such blatant markers of context for the encoding and decoding jobs to run smoothly.

\subsection{Coinage and Acceptability}

At this juncture it may be useful to think about the relation between abbreviations that save time and the entire question of the technical. It may help us to collect our thoughts around a passage from Cicero cited by Lefevere. Cicero, who translated many important Greek texts into Latin two thousand years ago, wrote about the neologisms he had to use: "By giving a Latin form to the text I had read, I could not only make use of the best expressions in common usage with us, but I could also coin new expressions, analogous to those used in Greek, and they are no less well received by our people, as long as they seemed appropriate" (De Oratore Book 1:35).

The Cicero passage is interesting for two reasons. First, of course, it is important that the problem of neologisms was noticed 
so early, in the context of the first high volume enterprise of translation - the Greek-to-Latin translation enterprise. However, and this is the second point, Cicero puts the problem in terms of coinage and public acceptability, not in terms of the use of the technical. In other words, the words he deal with do not strike him as being special words marking particular domains and deserving to be called technical or specialized vocabulary items whose use should in principle be controlled by the norms of a particular discipline. It is natural for Cicero to assume that the public alone controls the norms of all words. Thus the criterion of general acceptability to the community is decisive for him.

\subsection{Sublanguages and the Role of Specialists}

In contrast to that classical situation, we now live in a world where not only abbreviations proper, but even characteristic technical terms (such as Corpus Planning, a technical term in the language planning subfield of sociolinguistics) function as markers of a technical sublanguage subject to norms distinct from the general rules governing the language of the community. It is assumed, by specialist and layman alike, that the authority to discuss such sublanguages and to decide how their terms are to be appropriately used belongs to the specialists of the relevant disciplines or professions. The way we think about the matter is that those specialists spend all their time thinking about these things. Normal people do not have enough time to take the trouble of learning all the relevant details. So it is natural for ordinary members of the community to leave these technical matters to the specialists. This strategy, we ordinary people assume, saves us time and leaves us free to pursue our interests instead of getting caught in the specialized worries of this or that discipline at every step.

Notice, then, that the structure of a modern way of life, where the public saves time by allowing many differentiated subsocieties to develop and to worry about this or that set of technical details including the sublanguages in which those details need to be talked about, encourages the proliferation of technical jargon and abbreviations. For such an arrangement means that the members of each specialized subcommunity are encouraged to talk only to each other, not to people outside the boundaries of the discipline. They 
are also encouraged to save time for everybody, which means saving time for themselves also, if possible. This leads them to use abbreviations as much as they can, and to use ordinary terms in an unusual fashion which may be opaque to the uninitiated outsider but is understandable within the field. Used opaquely, even ordinary terms turn into abbreviations for entire collocations and ways of thinking that serve as common currency within a sublanguage but make no sense outside it. Hence the scene we have been describing in the earlier sections.

\subsection{Speed and Specialization}

Thus, the question of the technical revolves around notions like saving time and specialized subcommunities whose disciplinebound labour saves time for the community in general and also for the subcommunities themselves by using abbreviations or by deploying ordinary words in a way that turns them into abbreviatories of shorthand-like devices. This is not surprising. The logic of modern societies has to do with saving labour time and increasing productivity so that all members of society have a better life. The essence of high technology is to save more and more drudgery and thus release human energies for purposes that individuals are free to choose. One consequence is a mode of deployment of linguistic resources, like key words and abbreviations, which makes it possible to save communicative time, working rapidly and efficiently. Thus, speed and specialization go hand in hand.

\section{Names, Codes, and News}

\subsection{Form of Creativity and Naming}

We turn now to the connection between the speedspecialization complex just described and the point about novelty broached earlier. Why do modern societies desire to maximize the free action of individuals - a goal that leads them to resort to technologies that minimize the routine labour individuals have to do? Because we all want to release the energies of everybody's personal, expressive creativity in various domains. What form does creativity take? An original form, not bound by rigid traditions. Can technologies themselves turn into stifling rigidities? They can, if 
one does not frequently change the technological environment - a recycling which the technical world carries out in any case because of the pressures of competition and other familiar factors. Against the background of fast changing technologies, individuals trying to be creative have to express themselves clearly and distinctly, marking the originality of their creations. This is also true of persons who work as innovators in technical fields. Hence the general tendency to seek catchy titles for books, novel names for ideas and products, and other attention-grabbing identifiers of originality - not only in the world of advertising, but quite generally throughout all walks of life in a modern society. This is how one ends up getting a world featuring both abbreviations in the generalized sense and names in a sense we are about to examine in detail.

An original inventor seeking to safeguard his or her intellectual property rights normally proposes a unique label or designation identifying the creation as a new entity. This designation is notionally equivalent to the name given to a newborn baby to identify it. Even outside the legal context of patenting or otherwise securing rights over intellectual property, there is nowadays this general tendency to make up a brand name for something one has thought of, and an increased willingness on the part of our societies to uphold the rights of individuals to use and disseminate such names.

\subsection{Solution of Translation Problems}

The novelty involved in such naming or renaming takes somewhat different forms in different discourse domains. For example, natural and mathematical scientists tend to invent and use special words or word-combinations uniquely identifying phenomena and products. It is thus possible to keep up with their activities, by publishing periodically updated codifications - lists of generally accepted terms and what they designate. In contrast, scholars in the humanities and the social sciences prefer to reuse for their specialized purposes the expressions of ordinary language. A Lakatosian philosopher of science speaking of a research programme does not use the expression in the sense of a project at a university or institute seeking financial support from grant-giving 
bodies, but as a technical term for a dynamic cognitive matrix in which theories are formulated and considered. Sometimes it is unclear whether a term is being used technically, or is being highlighted in its ordinary sense which the thinker wants all readers to pay special attention to as in Ludwig Wittgenstein's ambiguous use of term, 'game', for example. Thus, translation problems for the natural and formal sciences can be solved in principle if the TL possesses or can invent the necessary new terms. In contrast, translation problems in human and social science disciplines are much more intractable in principle. A translator needs to be a sensitive reader capable of figuring out at which points the text makes a "move" and gives a particular locution the potency of technically or rigorously used wording, calling for special measures in the translated text.

\subsection{Code Book or Listing of Identifiers}

Abstracting away from these variations across discourse domains, one may speak in general of the formally innovative identifiers which authors use to mark their originality. For certain purposes, these identifiers may be regarded as names. Whether a society actually sets up terminological committees to keep track of these names or not, one may in principle imagine that all such names enter some sort of notional census listing, just as every newborn child gives rise to a new entry in a society's register which may or may not exist as a matter of physical fact. So imagined, the 'census listing' of identifiers is a codification of the naming process.

The idea of a code book, especially in the context of India as a culture whose code books were samhitaas, eternally valid codifications assumed to represent the structure of reality itself, may seem inappropriate for the type of national register we have in mind. Indeed, to the extent that name-like identifiers are created by individuals and need to be periodically renewed from generation to generation, the kind of codification which enables these identifiers to function as quasi-names does indeed differ from the code books of traditional societies. It is more useful to think of the national census listing of identifiers in terms of an imaginary permanent newspaper which reports every new emergence as a piece of news 
and whose files preserve these items for a reasonably long period over which they remain relevant. The newspaper is modern society's equivalent of the traditional samhitaa. Both the newspaper and the samhitaa codify. They both initiate and preserve the validity of the most significant designations (names) functioning in the speech community that they serve.

It is in the context of this nexus that names, codes, and news are thematically interdependent. In order to understand codes better, in the following section we shall look at the transition from samhitaatype codes to news-oriented codes - from ancientism to the valorization of modernity - in terms of the concepts of Archive and Arcade.

\section{Archives and Arcades}

\subsection{The Transition}

It is important to note that we are not speaking of a straightforward transition from archives to arcades. Let me explain what sort of distinction between two types of traditions is sought to be made by calling one kind 'archives" and the other kind 'arcades'. The question of archival traditions still looms large in the contemporary context. Likewise, although not quite at the same level of importance, there was something like an arcade issue in classical societies whenever an individual author put his stamp on a set of words and phrases marking his distinctive contribution to the culture. We are suggesting that archives are dominant in traditional cultures, while the arcade is a major force in modern textual formations and partly overshadows the archive.

While there is no transition from archives to arcades at the level of the very existence of these phenomena, we do wish to say that there is a transition from archival or samhitaa-type codes to arcadelike, news-oriented codes at the level of the notional registration of identifiers. This transition occurs in connection with a familiar shift in the distribution of social power.

\subsection{The Source of Authority}

The canonical source of authority in traditional societies was the king or some equivalent central figure. The king's registers, 
typically drawn from priesthood or some other body of professional intellectuals, represented his central authority and served as a collective register. This registrar embodied the right to preserve old identifiers and promulgate new ones. This authority was thus continuous with monarchical sovereignty in general. We see here the all pervasive centralization that marks the traditional model of social power. What held for all other forms of power also held for the power to identify. Sovereignty over all namings and all preservations of names vested in the king in principle, through the agency of the collective registrar responsible for maintaining the notional total register of all designations and identifiers.

\subsection{Changes in Codifiers}

Republican societies vest such authority in the public constituted as a senate. But this leaves the centralistic organization of power untouched if, as in republican Rome, an oligarchy capable of functioning consensually replaces the individual monarch but does not open up the space of power. Only the growth of democracy and of the socio-economic forces underpinning it brings about a crisis in the old regime. Out of such a crisis arises a modern structure of power. This new structure attaches great value to the innovative proposals emanating from non-central, non-ossified regions of the society. In order to maximize the dissemination and positive reception of such innovations, modern societies recast their rationality in a decentralizing direction. They permit the principle of continuity to remain in the form of law books and gazetteers and other standard codifications. But modern societies give newspapers and other periodical publications - including textbooks, which become increasingly tentative and updateable files rather than stable books in the old sense - nearly unlimited power to supplement and modify the content of the standard codifications.

It is here that the arcade where all proponents advertise their identifiers and products manages to overshadow the traditional archive. This overshadowing, while it does not destroy the archive's existence, decisively alters the type of codifications characteristic of the society. Books were the basic codifiers in traditional cultures which honoured old age. Newspapers and other journals become the basic codifier in modern cultures which cherish youth. 


\subsection{Records}

Suppose we use the term Records when we speak neutrally, spanning the range of societies from traditional to modern. Records may then be a set of archives with a tiny bit of arcade material, or alternatively a gallery of arcades overshadowing some surviving archives.

We may then say that any given term in a language - and, specifically, and neologism or other linguistic innovation - derives its particular semantic charge in the context of the echoes and counter echoes which the item evokes in the collection of Records available in that language. It is then immediately obvious that there will be an equivalent for the semantic charge of an item when one translates. For the TL Records will offer a different set of reverberations incapable of echoing the echoes and counter-echoes surrounding the original item in the SL. It follows that no neologism is strictly translatable, and that no TL neologism can strictly correspond to an ordinary SL term that calls for an innovation in the TL because there is no TL equivalent for that term. The point is of course trivially valid even for non-innovative or ordinary expressions. But it is quite forcefully so when we deal with terms and usages that are new and thus draw attention to their form and sense. If such attention-catching items are especially violative of the equivalence norms we expect in translation, then we have a problem that needs to be faced. And it interacts with the Translation Novelty Paradox that we started out with.

\subsection{The Contradiction}

This interaction has to do with the form that the problem takes in societies with a news-oriented codification system, where arcades have already become dominant or are fast becoming so. In these technicalized societies, neologisms live in an especially tight symbiosis with other ephemerally circulating items in the specific news world of a given region in a particular period. It would on the face of it seem completely impossible to transplant such a life form from one habitat to another. This gives us a spectacular version of the problem, leading us to ask how, under these conditions, the translation enterprise remains viable at all. For it would seem that 
much of a text would generally have to be fatally new, doing such violence to the TL codes and their expectations that the result of any translation attempt would inevitably be completely out of tune with audience assumptions and thus unintelligible, a non-text in the TL. This way of putting the problem is in touch with the Translation Novelty Paradox. For we must remember that, in the face of all this, the facts of the voluminous enterprise say to us, in the words of Galileo Galilei, e pur si muove ('and yet [the earth] moves [round the sun']. How is it that the impossible happens, and happens all the time? This is what makes the question a paradox, an apparent contradiction.

\subsection{Intertranslatability}

We can consider the issue first in the context of the group of languages forming the core of the successful translation enterprise in the modern period, the mutually translatable languages of the industrialized world. For English, French, German, Russian and other languages belonging to this group - not all of them genetically related, for Hungarian and Finnish fall with this core as well - the paradox can be resolved by nothing that the shared cultural archive puts all these languages on essentially the same map and enables them to overcome difficulties of transferring thematic reverberations during translation. These languages have mutual translation traditions. Their translators are used to coping with these difficulties.

For these intertranslatable languages, then, the problem dissolves to the extent that the interarchival gap has been bridged by an already existing cultural overlap and a large body of translations exerting a gravitational pull on new translated texts.

Intertranslatable languages are associated with industrialization. They have all moved into the news-oriented type of codification of the Records. Their arcades are in dialogue with each other. And their archives have long been rooted in shared histories. The difficulty is not acute in practice.

A much less tractable version of the issue arises for the languages of developing countries. To these we now turn. 


\section{Less Equipped Languages}

Many languages used in the developing countries have properties that make it useful to distinguish MELs (More Equipped - Languages) from LELs (Less Equipped Languages). They are in the process of developing registers and terminological repertories which will enable them to join the group intertranslatable modern languages, often with active state intervention of the type described as 'language planning'. For our immediate purposes, it is important to notice that in a LEL one expects large amounts of novelty on a routine basis, as most of the modern diction is supposed to sound new.

\subsection{Translationese}

This situation alters the premises of the Translation Novelty Paradox. In a LEL, there is no requirement that new entrants into the set of acceptable texts should exhibit any real continuity with the existing traditions so as to sound natural. Much can and will be written in a diction that will sound like 'translationese', without protest from the public. For the public wishes to catch up with the developed world, and accepts translationese as one of the costs of this endeavour.

The problem of translating from a MEL into a LEL, as far as neologisms are concerned, must be viewed in this context. Given the language planning enterprise and the presence of large amounts of routine innovation in such a TL, it becomes appropriate to describe the innovations in terms of a consciously and centrally authorized Archive. Such a language has no serious Arcade associated with it. It has too thick a historical layer of individual innovation admired and fraternally supported by other individuals the kind of layer that underwrites the Arcade in industrialized societies. All that a LEL can do is mimic the Arcade by using the resources that its new, rapidly created Archive of official coinages makes available.

\subsection{An Ad Hoc Solution}

The current global mingling of newspaper material (I am offering no specific treatment of the cyberspace nature of this 
mingling here) produces an ad hoc solution to this problem. The globalized consumerism of the middle class yields a rather low quality but highly disseminated cultural overlap which ensures a base line of mutual translatability in terms of widely known products and activities. Thanks to this base, even LELs can come up with viable short term equivalents for at least the journalistic input from MEL societies that has to be translated on a regular basis. Consumerism serves as a reference culture making most of the material intelligible. This solution amounts to a wholesale import of arcades. But it leaves the basic issues unaddressed. Linguistic innovation in the developing languages continues to be centrally authorized or monitored, because of the hierarchical structures of developing societies. Thus the Archive in these communities continues to overshadow the Arcade, producing a potentially explosive instability in the domains of innovation. Even the contemporary. wholesale import of the alien arcades is a centrally controlled and hierarchically sponsored initiative that can only reinforce the strength of the Archive's unity vis-à-vis the Arcade's multiplicity. Whether the forces of real creative innovation will succeed in finding a mode of expressing themselves, breaking through this authoritarian, elite-managed enterprise of copying foreign novelties - this issue concerns us all. Translators cannot afford to be neutral on this issue. For translation undertakes in general to minimize opacity and maximize transparency for the benefit of true self-expression and its dissemination. We must discharge this responsibility.

\subsection{Classification of Neologisms}

For our purposes, then, we need to adopt a classification of SL neologisms that keeps in view the appropriateness of dealing with them in particular ways when translating into a LEL. That is the burden of this section. Here is our classification:

(a) Name-based neologisms remain new as long as they retain the connection with what is perceived as a name. In this sense, 'stakhanovite' is a neologism even in our period, after the death of Stalinism and the near-oblivion of the Stakhanovite ideal of socialist workaholism. In contrast, "academic" is no longer a neologism or a name-based word, 
even though it did originate in a name when Plato founded his Academy. The treatment of (a)-type neologisms will depend on the method of translation adopted and the purpose that the translated text is intended for. It will also depend on the familiarity of the relevant name in the relevant LEL culture. Our task in translation studies is to alert the translator to types of recurrent problems, not to offer formulaic recipes. In general, one will want to treat "academic" as a timeless notion and "Stakhanovite" as a whimsically specific locution for 'hard-working labourer'. But a text focusing on Stalin's era will want to refer to biographical details about the heroically productive worker Stakhanov whom the Soviet system had held up as an example to be emulated. (b)Namemaking neologisms like 'glasnost', arising from occasions on which someone is able to recharge a normal word in a language and turn it into a name for a specific event or phenomenon, again pose a problem of judgment. If one is dealing with an event or phenomenon that has become or is capable of becoming common currency as a name in many countries, like glasnost, then it makes sense to transcribe the SL expression and treat it as a name in the TL. More often, one will have to find a sense equivalent in the target LEL and let the text do the work of showing the audience that the neologism is name like. A case of this sort is "generative grammar", a name-making neologism in English that has consistently been sense-translated, never transcribed, into other languages, with results ranging from sanjononi beakoron in Bangla to vyutpaadak vyaakaranx in Konkani. (c) Portmanteau neologisms like workaholic or affluenza sometimes start out as jokes, and during that period may elicit TL jokes from translators, like porisromaataal in Bangla blending porisrom 'work' with maataal 'drunk'. But words of this type which survive in the SL become standard pieces of natural language and have to be given more stable equivalents. Thus a second generation Bangla word for. workaholic would be something like kaajpaagol 'work-mad', an expression already used in ordinary texts. (d) Affixal neologisms formed by attaching affixes to existing bases and 
producing unusual combinations, like the word metadiscourse, can often be mimicked in a target LEL. Thus, if we agree to use baacokotaa in Bangla for 'discourse' and odhi for 'meta', as far as the rest of the terminological system is concerned, then it automatically follows that the appropriate expression for metadiscourse is odhibaacokotaa. If tomorrow a neologism "immigrantism" emerges to express the idea that all people are to be described as immigrants, then the existing Bangla expression obhibaasi 'immigrant' with the affix baad 'ism' will underwrite the new expression obhibassibaad. Affixes like meta and ism are easy. It is catch-all affixes like the prefix "de" that create problems. Recent writings in Bangla point up the difficulty of handling cases like deconstruction and decolonization. The natural equivalent for the former would be binirmaan. But one crucial terminologist was persuaded, apparently by a Sanskrit-erudite colleague, to avoid a collision with the Sanskrit word vinirmaanxa 'exquisite and careful construction'. Thus Bangla writings on the subject are divided between that terminologist's proposal abinirmaan and the other authors' choice binirmaan, a head-on collision within the language. and the mechanically derivable buponibeshon, for 'decolonization', using as it does the initial sequence bupo corresponding to vy-upa from Sanskrit, is phonologically opaque in Bangla and does not sound like a 'de'-word. One way out might be niruponibeshon, but onewould have to try it and see if the community accepts it. In general, there is no panacea that works for all cases. (e) Semantic neologisms, cases of existing expressions acquiring new senses, poses a problem if translators try to extend corresponding expressions into new semantic domains inexactly similar ways in the TL. One would be hard put to mimic the extension of the English adjective "explicit" from the articulate expression sense to the sense of film screen non-concealment of sexuality which became common the West in the sixties and seventies. The solution is perhaps not even to try. For 'explicit scene' one would then say in Bangla anaabrito drishsho (literally 'non-covered scene') and not 
bother about the unusual locution used to refer to that type of sequence in English. (f) Collocational neologisms, involving new combinations of words or new senses for combinations already available, often pose problems for the translator if the TL already has another set of connotations for what would be the normal TL equivalent. Thus, if the German Greens lead a revolution, drastically changing the politics and culture of Central Europe, it will not be appropriate to describe such an event as a green revolution in Indian English or as a shobuj biplab in Bangla, for these terms are already laden with the historical specificity of the revolutionary introduction of high yield seed varieties in Indian agriculture in the sixties. Perhaps an expression like the Green's Revolution or shobujder biplab will manage to convey the sense of a revolution led by the Greens. (g) Acronyms and abbreviations proper, to turn at last to the topic that has crucially shaped our approach to the overall issue, often pose a difficulty at the very first stage of the translator's attempt to handle a text, in that we often fail to understand a new abbreviation or acronym even in a domain we may otherwise be familiar with. It is important to keep in touch with handbooks, periodicals, and the community of colleagues in the domain so that one can get help in deciphering these unidentified frustrating objects.

The next question - how to present the material in the TL - has to do with the habits one's audience has and the new ones you want them to acquire. Bangla speakers do use acronyms, as in the nineteenth century saint Sri Ramakrishna saying as he comes out of a gathering where one has to leave one's footwear at the door: ju aa naa ge?, short for juto aache naa geche? 'Are our sandals still there or are they gone?' But one prefers to refer to WHO 'World Health Organization' in Bangla as "Bishsho Shaastho Shangsthaa", not by using an acronym like "Bi-shshaa-sh" which would converge with the homophonous word "bishshaash" which means 'faith, belief', the kind of convergence Westerners seem to like for their acronyms. Translators who wish to strive for transparent communication need to respect such preferences in the TL 
community, unless they know of any reasons for initializing a drastic reform in the audience's habits.

\section{Changing the TL}

The single most potent source of changes in vocabulary and diction in the developing languages in the neologisms produced and reproduced by journalists functioning as translators. Working under high pressure, these translators who often have no translation training and certainly no knowledge of language planning theory are forced to respond immediately to the problem of finding equivalents for English expressions pouring into the newsroom, expressions for which Less Equipped Languages have no standard equivalents.

As education becomes a powerful instrument of social change, a second important source will be school and college textbooks in LELs, supplemented by other academic publications. Again, the strategy of authors producing such material is often to translate from English.

Journalists, educators, and other less powerful translators will decide for each community of this sort to what extent existing styles and registers will change to adjust to the prevalent patterns of innovations in the MELs. The translators cannot, of course, make any unilateral moves. Their proposals are at the disposal of the public which accepts and rejects them along unpredictable lines (but see Anita, forthcoming). It is thus a consultative, communitywide process that ensures real application of rationality norms in term planning.

The pressures of modern life described above which make abbreviation a powerful general tendency also encourage such translators, and the public which has a filtering effect on their work, to import acronyms like UNICEF and UNESCO directly into other languages, especially LELs. Likewise, scientific and technical notations consisting of (or incorporating) various types of abbreviation tends to be carried over into LELs with little or no modification.

Under these conditions, it is an open question whether terminological committees and institutions of the sort sponsored by 
typical third world governments and advised by linguists and professional translators can do anything worthwhile by creating vocabularies in the usual fashion. The work done by such bodies exerts a great influence on the diction of journalists and textbook writers, and through them on the general public, despite various filter-effects. In other words, term planning of the committee variety does help. However, such planned vocabularies cannot cope with the fast pace of change and innovation in many domains. Committees work slowly and on the basis of consensus. They tend to put senior persons from humanities disciplines and literature backgrounds in key positions. Such figures impose their overall taste on the output of these committees. This taste is consistently conservative and favours the archive approach, tending to neglect or not even to understand the imperative of the arcades of our modern societies.

Under these conditions, since there is no known way to change the composition of term planning committees or the style of the humanities disciplines in LEL speech communities, and since therefore the standard handbooks ultimately controlled by such authorities will act as a brake on the development of a modern diction, translators need to learn how to use their own judgment and make spontaneous decisions that strengthen the forces of innovation in LELs.

\section{References}

Bassnett, Susan. \& Lefevere, Andre (eds.) 1990 Translation, History, and Culture. London / New York: Pinter.

Bassnett-McGuire, Susan 1980 Translation Studies. London: Methuen.

Lefevere, Andre 1990 Translation: Its Genealogy in the West. In Bassnett and Lefevere (eds.) 14-28.

Ravanam, Anita (forthcoming). Three Models of Lexical Codification. Forthcoming in The Yearbook of South Asian Languages and Linguistics. 